\title{
Les ouvriers dans les manifestations révolutionnaires à Nantes en 1789-1791 : vers une identité collective?
}

Workers in the revolutionary movements in Nantes in 1789-1791: the making of a collective identity?

\section{Samuel Guicheteau}

\section{(2) OpenEdition Journals}

\author{
Édition électronique \\ URL : https://journals.openedition.org/ahrf/11476 \\ DOI : 10.4000/ahrf.11476 \\ ISSN : $1952-403 X$
}

Éditeur :

Armand Colin, Société des études robespierristes

\section{Édition imprimée}

Date de publication : 1 janvier 2010

Pagination : 75-95

ISSN : 0003-4436

Référence électronique

Samuel Guicheteau, «Les ouvriers dans les manifestations révolutionnaires à Nantes en 1789-1791: vers une identité collective? », Annales historiques de la Révolution française [En ligne], 359 | janviermars 2010, mis en ligne le 01 mars 2013, consulté le 23 avril 2022. URL : http:// journals.openedition.org/ahrf/11476 ; DOI : https://doi.org/10.4000/ahrf.11476 


\section{LES OUVRIERS DANS LES MANIFESTATIONS RÉVOLUTIONNAIRES À NANTES EN 1789-1791 : VERS UNE IDENTITÉ COLLECTIVE?}

Durant la Révolution, les ouvriers nantais se mobilisent lors des émeutes frumentaires soulevant l'ensemble du peuple et mènent aussi des luttes particulières, usant de pratiques largement populaires ou spécifiquement ouvrières. Ces différentes manifestations favorisent l'articulation du social et du politique, donc la maturation d'une conscience révolutionnaire. Si l'expérience révolutionnaire des ouvriers s'avère remarquable et si certains de ses aspects originaux peuvent renforcer leur identité commune, elle ne donne pas nécessairement naissance chez eux à conscience collective et spécifique.

Mots-clés : ouvriers, identité, émeutes, pratiques, droits, conscience sociale.

Dans sa contribution au colloque Vers un ordre bourgeois? Révolution française et changement social, Haïm Burstin écrit que la Révolution ne provoque pas brutalement l'homogénéisation sociale de la bourgeoisie, mais amène les bourgeois à élaborer, dans le feu de la dynamique révolutionnaire, "la conscience d'être un sujet politique autonome », sans que pour autant ils adoptent des comportements politiques similaires. De plus, il suggère qu' « il en est de même pour [d'autres groupes, notamment] les couches populaires qui prennent souvent conscience de leur identité sociale et de leur autonomie potentielle au cours même des luttes politiques, revendicatives ou sociales $»^{1}$. Notre contribution vise à

(1) Haïm Burstin, « Bourgeois et peuple dans les luttes révolutionnaires parisiennes », dans Jean-Pierre JESSENNE (dir.), Vers un ordre bourgeois? Révolution française et changement social, Actes du Symposium international de Villeneuve-d'Ascq, Rennes, PUR, 2007, p. 175-176. 
expérimenter la transposition de cette perspective, non à l'ensemble des couches populaires, mais aux seuls ouvriers. $\mathrm{Si}$, au XVIII ${ }^{\mathrm{e}}$ siècle, l'unique mention du métier comme l'ambiguïté du terme ouvrier masquent la condition sociale exacte, il est néanmoins possible d'identifier des ouvriers : d'une part, la condition ouvrière est parfois précisée grâce à l'adjonction au métier des termes « compagnon » ou « journalier »; d'autre part, certaines sources, en particulier policières et judiciaires, livrent la condition sociale exacte. Nous n'emploierons nous-même le terme ouvrier que pour désigner des salariés et des chambrelans ${ }^{2}$.

À la veille de la Révolution française, bourgeois et ouvriers présentent, sur le plan social, des traits comparables : une réelle diversité s'accompagne de l'existence de caractéristiques communes. Il nous semble que l'existence de celles-ci permet une appréhension globale des ouvriers qui, à la fin du XVIII ${ }^{\mathrm{e}}$ siècle, sont de plus en plus nombreux à Nantes, où les activités industrielles sont importantes, multiples et en plein essor ${ }^{3}$. Bien sûr, le monde ouvrier nantais présente une importante hétérogénéité. Il est même traversé par des tensions et des césures. Il n'est nullement animé par une conscience collective, dans la mesure où les ouvriers cultivent une conscience essentiellement professionnelle. De plus, les ouvriers restent insérés dans l'ensemble du peuple, au sein duquel ils côtoient les maîtres artisans et les travailleurs employés hors de l'industrie; ces catégories populaires partagent des pratiques et des valeurs et sont liées par diverses relations, de voisinage ou de famille notamment. Néanmoins, il nous semble que les ouvriers possèdent aussi une identité propre, forgée par eux-mêmes au travail et fondée sur la qualification et son exaltation d'une part, et sur l'attachement à l'autonomie d'autre part. Cette identité 4 est commune à la plupart d'entre eux, par-delà la diversité des cadres de travail, des niveaux de qualification (le sentiment de détenir un savoir-faire est essentiel, comme le montre le cas des portefaix) et des situations économiques (salarié ou façonnier). En outre, tous les ouvriers sont touchés, certes de manières et à des degrés variés, par l'industrialisation, entendue comme un processus général de mutations économiques, sociales et culturelles. Les ouvriers nantais ne sont donc pas saisis par la

(2) La déchéance de certains maîtres, isolés, pauvres et dépendants, n'autorise pas à les confondre avec les ouvriers.

(3) Samuel Guicheteau, La Révolution des ouvriers nantais. Mutation économique, identité sociale et dynamique révolutionnaire (1740-1815), Rennes, PUR, 2008.

(4) Pour nous, l'identité associe donc à des caractéristiques socio-économiques, des aspects socio-culturels, c'est-à-dire des pratiques, des valeurs et une conscience (qu'elle soit populaire, ouvrière ou professionnelle). 
Révolution française comme un «matériau humain brut $»^{5}$. Au contraire, leur participation à celle-ci est sans doute marquée - en partie - tant par leur identité sociale et culturelle que par les tensions inhérentes à l'industrialisation.

Par conséquent, il est possible d'évoquer une expérience ouvrière de la Révolution française. Mais il faut aussitôt préciser que, dans la mesure où les ouvriers ne forment pas un groupe homogène et où ils restent insérés dans l'ensemble du peuple, leur expérience révolutionnaire ne se réduit ni à des questions ni à des modalités spécifiquement ouvrières, mais s'inscrit aussi plus largement dans l'ensemble de la participation populaire à la Révolution, tant sur le plan social (comme le montre le caractère essentiel des subsistances) que sur le plan politique (comme en témoignent le rythme et certaines modalités de la politisation). Plusieurs questions s'articulent donc : comment se nouent le social et le politique à travers une participation multiforme des ouvriers à la Révolution? Comment mûrit leur conscience révolutionnaire, issue précisément de l'articulation du social et du politique, et quel est son éventuel effet sur leur identité sociale? Pour traiter ces questions, nous nous intéresserons aux émeutes qui éclatent à Nantes au début de la Révolution française et au cours desquelles s'affirment la conscience sociale et la politisation. Nous pourrons observer l'entrecroisement de motifs largement populaires et spécifiquement ouvriers, la circulation des pratiques et des valeurs tant ouvrières que populaires, ainsi que la maturation révolutionnaire des ouvriers.

\section{Émeutes et émotions à Nantes : les ouvriers dans les manifestations populaires}

Alors qu'elles avaient disparu depuis longtemps, des émeutes éclatent de nouveau à Nantes durant les premières années de la Révolution ${ }^{6}$.

(5) Edward Thompson, La formation de la classe ouvrière anglaise, Paris, Seuil, 1988, p. 74 (la formule s'applique à la Révolution industrielle). E. Thompson souligne aussi que la contribution des ouvriers au façonnement de leur propre identité est essentielle.

(6) L'exploitation des archives judiciaires et l'étude des délibérations municipales apportent de précieux éclairages sur ces émeutes, qui, comme de nombreux épisodes de la Révolution française à Nantes, n'ont longtemps été connues qu'à travers les récits livrés par les historiens locaux du XIX ${ }^{\mathrm{e}}$ siècle : Ange GuÉPIn, Histoire de Nantes, Nantes, Mellinet-Sébire, 1839; Camille Mellinet, La Commune et la Milice de Nantes, Nantes, Mellinet, 1841-1843, t. 6 à 10. 


\section{«Il nous faut du pain », mais aussi du travail}

Ces émeutes éclatent pour des motifs sociaux, au premier rang desquels apparaissent, bien sûr, les subsistances : «Il nous faut du pain » ${ }^{7}$, s'exclame l'un des émeutiers de janvier 1789; « du pain ou de l'ouvrage ", précisent d'autres émeutiers ${ }^{8}$. Cette émeute éclate le 8 janvier lorsque 500 à 600 Nantais envahissent la mairie pour se plaindre de la disette et dénoncer l'accaparement. Le meneur de l'émeute ayant « dit qu'il connaissoit une maison où $[\ldots]$ l'on receloit une grande quantité de sacs de farine $»^{9}$, un échevin accompagne les émeutiers pour la perquisitionner. Cette maison est saccagée, l'échevin molesté, puis l'hôtel de ville de nouveau envahi par un cortège fort désormais de « deux milles personnes de tous sexes $\gg^{10}$, si bien que la municipalité baisse le prix du pain. Le 25 mai 1790, la foire nantaise est troublée par une émeute traditionnellement présentée comme le fait de ruraux, hostiles au droit d'octroi et manipulés par les aristocrates et les prêtres contre-révolutionnaires. Si ce motif comme le rôle de boutefeu de ces acteurs sont confirmés par la procédure judiciaire ${ }^{11}$, celle-ci révèle, d'une part, que les ruraux se montrent moins violents que les nombreux travailleurs citadins qui participent à l'émeute, et, d'autre part, que le pain n'est pas absent des préoccupations des émeutiers. D'ailleurs, le 31 mai, son prix est baissé pour les pauvres ${ }^{12}$.

Le 5 septembre 1791, éclate la dernière émeute de la décennie révolutionnaire. Un rassemblement de plusieurs centaines de personnes se présente de nouveau devant la mairie pour dénoncer la perte que leur fait subir l'usage des cartes de la caisse patriotique ${ }^{13}$ et réclamer leur suppression. Tandis que le maire s'adresse à la foule, celle-ci tente d'envahir l'hôtel de ville : la loi martiale est alors proclamée, ce qui n'empêche pas la multiplication des affrontements au cours de la journée. Deux mois plus tard, la municipalité est alertée de la préparation par les compagnons du devoir « d'un attroupement de plus de trois mille ouvriers de tous états dans la

(7) AD Loire-Atlantique, B 8746-IV, déclaration de l'avocat du roi au présidial de Nantes, 10 janvier 1789 .

(8) AM Nantes, FF 289, procès-verbal de police, 7 janvier 1789.

(9) AD Loire-Atlantique, B 8746-IV, déclaration de l'avocat du roi...

(10) AM Nantes, FF 289, procès-verbal de police, 8 janvier 1789.

(11) AM Nantes, I 3-D 99.

(12) AM Nantes, 1-D-4, registre de délibérations municipales, $\mathrm{f}^{\circ} 21$.

(13) Comme les assignats n'existent encore que sous la forme de très grosses coupures, les municipalités créent des caisses patriotiques qui émettent de petites coupures. Un ouvrier menuisier explique que « sur une carte de douze francs, on vouloit lui retenir dix sols de bénéfice sur huit francs qu'il avoit à payer » (AD Loire-Atlantique, L 1439, interrogatoire d'A. Leroux, 8 septembre 1791). 
partie des ponts, [qui] ne demandent pas moins que l'abolition des billets de la caisse patriotique, ou sinon [qui] promettent de faire la police euxmêmes $»^{14}$. Un compagnon tonnelier est soupçonné d'avoir dit à un tisserand « qu'ils étoient 1800 compagnons du devoir, tous déterminés » ${ }^{15}$. En fait, si le rejet de ces billets persiste, l'assemblée projetée par les compagnons ne vise pas à relancer l'émeute, mais à consacrer la fondation d'un compagnonnage chez les maréchaux-ferrants nantais ${ }^{16}$.

En 1789-1791 éclatent aussi plusieurs émotions de moindre envergure. À l'automne 1789, la disette suscite une certaine agitation. Deux Nantais sont arrêtés pour avoir distribué des billets appelant à des rassemblements et à des perquisitions ${ }^{17}$. Un portefaix est accusé d'avoir proclamé qu'il fallait «faire une révolte pour aller chez les boulangers prendre du pain $»^{18}$. Ces deux procédures témoignent d'une agitation plus vaste, suffisamment forte pour que le colonel d'Hervilly, chef de corps du régiment cantonné à Nantes, tente d'enrôler le meneur de l'émeute de janvier dans un complot aristocratique, dont le but serait d'attiser la colère populaire contre les autorités issues de la révolution municipale ${ }^{19}$. L'année suivante, la crainte de la disette pousse des femmes à empêcher des convois de blé de quitter Nantes, initiative dont elles informent aussitôt la mairie; des perquisitions sont ensuite menées chez des individus soupçonnés d'accaparement ${ }^{20}$.

Par ailleurs, le 24 février 1790, les ouvriers maçons adressent à la municipalité une pétition dans laquelle ils sollicitent son intervention pour améliorer leurs conditions de travail. Si elle leur donne raison sur plusieurs points (mais pas sur le salaire, ni sur la durée de l'embauche), elle interdit dès le lendemain les rassemblements d'ouvriers qui semblent se multiplier et pourraient prendre une tournure séditieuse ${ }^{21}$. D'autre part, en octobre, puis en décembre 1790, le domicile de l'édile responsable de l'atelier municipal de charité est assailli par les ouvriers qui y sont

(14) AM Nantes, 1-D-5, f 99 , 24 novembre 1791.

(15) AD Loire-Atlantique, L 2073, lettre de L. Saget au maire, 24 novembre 1791.

(16) Voir infra.

(17) AD Loire-Atlantique, B 8745-VI, interrogatoire de J. Giraud, 24 septembre 1789.

(18) AD Loire-Atlantique, B 8745-VI, déposition de P. Monsacré, 13 octobre 1789.

(19) Le colonel d'Hervilly propose de « faire une révolte : nous rétablirons les nobles dans leurs droits, et le peuple ne mangera plus de mauvais pain » (AD Loire-Atlantique, B 8746-IV, déposition de D. Kervégan, maire de Nantes, 2 octobre 1789). Après son entrevue avec ce colonel, le meneur de l'émeute de janvier alerte les autorités (interrogatoire de J. Lédat, 28 septembre 1789).

(20) AM Nantes, 1-D-3, f 115 et 119,13 et 16 septembre 1790.

(21) AM Nantes, F 7-C 7-D 1. 
employés. L'annonce par la municipalité du renvoi des étrangers ${ }^{22}$, au nom de la priorité qui revient aux Nantais, provoque un premier rassemblement fort de trois cents ouvriers. Deux mois plus tard, cinquante ouvriers se réunissent à nouveau et, comme en octobre, menacent l'édile et violent son domicile ${ }^{23}$. Si, à l'instar de l'assemblée des compagnonnages prévue en novembre 1791, ces actions ne mobilisent que des ouvriers, ces derniers sont également fortement présents dans les émeutes de subsistances qui, elles, sont menées par l'ensemble du peuple.

Des « gens de tous états $»^{24}$

Plutôt qu'à la hiérarchie des rangs dans la société d'Ancien Régime, cette formule renvoie probablement à la diversité des métiers, repérables à leurs habits et à leurs outils. Ainsi, le 25 mai 1790, les « bonnets, veste et tabliers $\rangle^{25}$ trahissent la présence de citadins parmi les émeutiers. Un garçon charron indique que, le 5 septembre 1791, il a été enrôlé dans l'émeute par « une troupe de maçons, tailleurs de pierres et manœuvres, autant qu'il put les distinguer à leurs vêtements »; ce même jour, un ouvrier marbrier reconnaît les « ouvriers de navires [à] leurs vêtements gaudronnés $»^{26}$. Mais les commissaires de police sont aussi sensibles à la présence parmi les émeutiers de janvier 1789 de quelques individus « assez bien vestus $»^{27}$.

Les quinze prévenus poursuivis pour leur participation à l'émeute de janvier 1789 appartiennent tous au peuple : certains sont de petits marchands (un marchand-sabotier, un mercier), d'autres des salariés (deux portefaix, un charpentier, un maçon ${ }^{28}$, ainsi que trois journalières, une tailleuse et une garde-malade); enfin, dans quelques cas, nous ignorons leur condition sociale exacte (pâtissier, tonnelier, cordonnier). Le 25 mai 1790, au premier rang des émeutiers apparaissent des « ouvriers artisans $»^{29}$, formule particulièrement ambiguë. En fait, parmi les seize

(22) AM Nantes, 1-D-3, f 133,9 octobre 1790.

(23) AD Loire-Atlantique, L 1440, décembre 1790.

(24) AM Nantes, FF 289, procès-verbal des échevins, 8 janvier 1789.

(25) AM Nantes, I 3-D 99, déposition d'A. Debeluc, 26 mai 1790.

(26) AD Loire-Atlantique, L 1439, interrogatoires de C. Aubert et de J. Chauveau, 8 septembre 1791

(27) AM Nantes, FF 289, procès-verbal de police, 8 janvier 1789.

(28) La procédure judiciaire signale la condition d'ouvrier du maçon meneur de l'émeute. Le charpentier est présenté comme journalier dans le registre de la capitation nantaise de 1789 (AD Loire-Atlantique, B 3530).

(29) AM Nantes, I 3-D 99, déposition d'A. Debeluc, 26 mai 1790. 
personnes interrogées au lendemain de l'émeute, figurent quatre ruraux (tous libérés) et douze Nantais, notamment cinq ouvriers ${ }^{30}$ et six domestiques (l'inculpation de quatre d'entre eux témoigne de l'action de boutefeu jouée par leurs maîtres, prêtres ou aristocrates). Parmi les vingt-et-un prévenus figurent cette fois huit bouchers, sans doute intéressés par la suppression du droit d'octroi. Plusieurs témoins indiquent qu'ils ont contribué de manière décisive au déclenchement de l'émeute; l'un d'eux «a entendu dire [que] cette émeute avoit été causée par les bouchers, garçons cotonniers et autres ouvriers $\|^{31}$. L'émeute du 5 septembre 1791, quant à elle, mobilise « la classe des journaliers $»^{32}$. Plus précisément, les ouvriers du port y jouent un rôle important : dix-sept prévenus sur soixante-deux travaillent au port ${ }^{33}$; vingt-six autres, parmi lesquels dix-sept sont certainement des ouvriers, exercent une autre activité industrielle ${ }^{34}$; on ignore la condition sociale exacte de la plupart des autres travailleurs interrogés au lendemain de l'émeute. Enfin, les deux Nantais poursuivis à l'automne 1789 pour avoir diffusé des billets appelant à la mobilisation contre la disette, sont cafetier et maitre tailleur d'habits; mais, capité à une livre en 1789 , ce dernier est sans doute un travailleur isolé.

Les émeutes mobilisent donc l'ensemble du peuple nantais. Les ouvriers y jouent souvent un rôle décisif: en janvier 1789, les maçons dirigent l'émeute ${ }^{35}$; en septembre 1791, ce sont les ouvriers du port qui en prennent la tête.

\section{Pratiques et valeurs des émeutiers}

Par leurs motifs comme par leurs acteurs, les émeutes se révèlent essentiellement populaires. Si les ouvriers agissent parmi l'ensemble du peuple, peut-être recourent-ils néanmoins à des pratiques spécifiques. L'exploration plus profonde de la complexité sociale de ces manifestations requiert en fait une approche identitaire.

(30) Parmi lesquels un bonnetier et un tailleur d'habits qui seront inculpés.

(31) AM Nantes, I 3-D 99, déposition de F. Cochet, 29 mai 1790.

(32) Le terme « journalier » désigne ici plutôt l'ensemble des salariés que les seuls ouvriers non-qualifiés (AM Nantes, 1-D-5, $\mathrm{f}^{\mathrm{o}} 68,5$ septembre 1791).

(33) Ils sont charpentiers de navire, tonneliers ou portefaix. De plus, cinq des huit délégués reçus par le maire, dont le métier est connu, sont des ouvriers de la construction navale (AM Nantes, $\left.1-\mathrm{D}-5, \mathrm{f}^{\circ} 65\right)$

(34) Il s'agit notamment de travailleurs du bâtiment.

(35) Outre le meneur Joseph Lédat, on relève notamment l'action de trois frères également maçons. 
«Si ces messieurs ne venoient à leurs secours, ils formeroient une révolte générale $»^{36}$

Comment naissent les émeutes? En janvier 1789, l'émeute naît de plusieurs rassemblements successifs : le premier se tient le 7 devant le domicile du sous-maire; le lendemain matin, le meneur de l'émeute s'écrie que «c'est aujourd'hui qu'il faut que le pot crève ${ }^{37}$; ensuite, l'émeute se forme dans l'après-midi et envahit la mairie à deux reprises; enfin, le 9 un nouveau rassemblement parcourt les boulangeries. Au cours de cette émeute apparaissent deux types de mobilisation : d'une part, le rôle primordial que jouent les maçons témoigne d'une mise en œuvre de la sociabilité professionnelle; d'autre part, le fait qu'une boulangerie soit pillée par des voisins révèle une mobilisation à l'échelle du quartier. Dans les deux cas, le rassemblement de la foule s'opère selon une sociabilité et une solidarité qui lui préexistent ${ }^{38}$. La mobilisation professionnelle peut aussi mettre en branle des ouvriers réunis sur un même lieu de travail, ceux de l'atelier municipal en octobre-décembre 1790, puis ceux des chantiers navals le 5 septembre 1791. Ce jour là, partie des chantiers « du bas de la Fosse, une troupe de trois à quatre cens hommes » remonte la Loire; puis, « chemin faisant, ils se sont prodigieusement grossis par les ouvriers qu'ils ont rencontrés dans leur chemin travaillant à divers navires, chantiers et atteliers, constructions de maisons et déchargement de gabarres $\gg{ }^{39}$. Cette mobilisation professionnelle se retrouve aussi chez les bouchers le 25 mai 1790. Par ailleurs, en septembre 1790, ce sont des femmes du quartier des Ponts qui arrêtent les convois de grains. Les modalités de formation des émeutes relèvent donc de la sociabilité de quartier comme de métier. Si celle-ci ne concerne pas que les ouvriers, ils peuvent également être pris dans la mobilisation de voisinage.

Les émeutiers recourent à la violence. Des domiciles sont visés par certains rassemblements, celui du sous-maire le 7 janvier 1789, puis celui de l'édile chargé de l'atelier de charité en octobre-décembre 1790. Dans ce second cas, le domicile est même violé, comme ceux soumis à des perquisitions lors des troubles de subsistances, en janvier 1789, puis en septembre 1790. Des menaces sont proférées. Le 8 janvier 1789, les

(36) AM Nantes, FF 289, procès-verbal de police, 7 janvier 1789.

(37) AD Loire-Atlantique, B 8746-IV, interrogatoire de J. Lédat, 28 septembre 1789.

(38) Georges LefEBvre, "Foules révolutionnaires », Annales historiques de la Révolution française, 1934, p. 1-26.

(39) AD Loire-Atlantique, L 1439, interrogatoires de J. Chauveau et d'E. Gouillard, 8 septembre 1791 
émeutiers adressent à l'échevin qui les accompagne « les plus affreuses menaces, comme de le jetter dans le feu ou dans l'eau et de l'assomer à coups de bâtons $»^{40}$. Le 6 décembre 1790, les ouvriers de l'atelier de charité proclament que si l'édile qui en est responsable, «n'avait pas la force de porter sa teste, ils la lui porteraient eux-mêmes $»^{41}$. Enfin, la violence éclate. Le 25 mai 1790, le bureau d'octroi est saccagé. Le 8 janvier 1789, l'échevin emmené par les émeutiers revient à l'hôtel de ville «pâle, défiguré, ses cheveux dans le dernier désordre, [après] avoir été indignement maltraitté par le peuple, foulé aux pieds [...], et ayant le visage ensanglanté $»^{42}$. En 1790 et 1791, des affrontements opposent gardes nationaux et émeutiers. En septembre 1791, le procureur de la commune souligne «qu'il n'est aucun quartier où on ait attaqué, désarmé et maltraité des gardes nationales $»^{43}$. S'y ajoutent des défis : ainsi, alors qu'il tente d'entrer dans la mairie, un émeutier se heurte à « un des gardes nationaux [qui] l'avoit menacé de le bourrer avec sa bayonnette, mais il avoit [alors] découvert sa poitrine et l'avoit défié d'exécuter ses menaces $\rangle^{44}$.

Le recours à la violence s'explique par le sentiment de légitimité de la revendication qu'éprouve le peuple nantais. Face à la disette ou à l'agiotage, ce dernier attend des autorités qu'elles assument leur responsabilité. C'est pourquoi les rassemblements se portent à l'hôtel de ville, lors des émeutes de 1789 et 1791 comme lors de l'agitation de l'automne $1789^{45}$ et des troubles de septembre 1790. La défaillance des autorités libère le peuple de son devoir d'obéissance. Dès le 7 janvier 1789, le meneur de l'émeute déclare « demander justice, disant qu'ils se la feraient eux-mêmes si on la leur refusait $\gg^{46}$. Cette exigence et cette menace sont répétées le lendemain à l'hôtel de ville ${ }^{47}$. La même attitude se retrouve le 5 septembre 1791 : après avoir « déclaré que toute espèce de révolte était loin de leurs intentions », les délégués reçus par la municipalité, frustrés de ne pas obtenir la satisfaction immédiate de leur revendication, auraient annoncé « que si on ne leur accord[ait] pas pleinement ce qu'ils demand[ai]ent, ils ces-

(40) AD Loire-Atlantique, B 8746-IV, déclaration de l'avocat du roi...

(41) AD Loire-Atlantique, L 1440, déposition de F. Durong, 9 décembre 1790.

(42) AM Nantes, FF 289, procès-verbal des échevins, 8 janvier 1789.

(43) AM Nantes, FF 105, registre des audiences de police, f 24,7 septembre 1791.

(44) AD Loire-Atlantique, L 1439, déposition d'E. Beaudouin, 17 septembre 1791.

(45) Le maître tailleur d'habits interrogé pour avoir appelé, avec un complice, à un rassemblement « répond [qu'] ils avoient comme arrêté entre eux de faire une requête et de la présenter au maire de cette ville pour l'engager de faire diminuer le prix du pain » (AD Loire-Atlantique, B 8745VI, interrogatoire de R. Lebrun, 24 septembre 1789).

(46) AD Loire-Atlantique, B 8746-IV, interrogatoire de J. Lédat.

(47) AM Nantes, FF 289, procès-verbal de police, 8 janvier 1789. 
ser[aie]nt de travailler et se procurer[aie]nt de quoi vivre par le pillage $\iota^{48}$. Tout retard est assimilé à une manœuvre dilatoire, voire à un inacceptable déni de la légitimité de la revendication. Le 8 janvier 1789 , de retour à l'hôtel de ville, les émeutiers ne se retirent qu'une fois adoptée la nouvelle police du pain : «Après avoir soutenu pendant une heure un choc si furieux contre un peuple révolté qui n'entendait aucune raison », les échevins sont contraints de céder ${ }^{49}$. De même, le 5 septembre 1791, les émeutiers refusent d'entendre le discours du maire : à peine a-t-il ouvert la bouche, que l'un d'eux « a crié que l'on n'écouterait rien, ajoutant avec violence : point de cartes, point de cartes $\rangle^{50}$.

La défaillance des autorités pousse le peuple à agir directement, comme le montre un billet distribué en septembre 1789: «Assemblons peuple et bon citoyens nantais pour faire diminuer le pin, alons visiter les greinier sans tarder $\rangle^{51}$. L'année suivante, les femmes qui ont arrêté des convois de blé, ne les laissent pas repartir, en dépit des propos rassurants du maire ${ }^{52}$. En revanche, une fois la revendication satisfaite, les autorités peuvent retrouver leur légitimité, comme en témoigne l'attitude des émeutiers qui, le 9 janvier 1789, s'exclament « vive le roi ! vive monsieur Desplantes! vive monsieur Geslin qui nous a fait diminuer le pain $\rangle^{53}$. En effet, l'acquiescement des autorités restaure le contrat frumentaire et politique qui les lie au peuple. Ainsi, le recours à la violence par les émeutiers s'explique par leur sentiment de sa légitimité, issue de leur relation contractuelle avec les autorités, qui s'inscrit elle-même dans la perspective de l'économie morale ${ }^{54}$. L'attachement populaire à celle-ci se retrouve dans la pratique de la taxation. Accusé d'avoir pillé une boulangerie, le meneur de l'émeute de 1789 réplique qu'il s'est contenté d'imposer le respect du nouveau tarif : visitant le 9 janvier les boulangeries, les émeutiers « rencontrèrent un couvreur qui leur dit avoir quatre enfants et point de pain chez lui, qu'il venait de chez un boulanger [...] qui avait

(48) AM Nantes, 1-D-5, $\mathrm{f}^{\mathrm{o}} 65$.

(49) AM Nantes, FF 289, procès-verbal des échevins, 8 janvier 1789.

(50) AM Nantes, 1-D-5, f ${ }^{\circ} 65$.

(51) AD Loire-Atlantique, B 8745-VI, interrogatoire de J. Giraud, 24 septembre 1789.

(52) AM Nantes, 1-D-3, f 116,14 septembre 1790.

(53) AD Loire-Atlantique, B 8746-IV, interrogatoire de J. Lédat. Les personnages cités sont le sous-maire et l'échevin molesté.

(54) Edward Thомpson, «L'économie morale de la foule dans l'Angleterre du XVIII" siècle ", dans Florence GaUthier, Guy-Robert IKNI, La guerre du blé au XVIII siècle. La critique populaire contre le libéralisme économique au XVIII ${ }^{e}$ siècle, Montreuil, éd. de la Passion, 1988, p. 31-92 (1971); Dominique Margairaz, Philippe Minard, « Marché des subsistances et économie morale : ce que “taxer” veut dire », Annales historiques de la Révolution française, 2008-2, p. 53-99. 
voulu lui en vendre un plus cher que le prix de la police »; ils se portèrent chez ce boulanger récalcitrant et « ce couvreur prit [alors] un pain de douze livres, pour lequel ils lui firent compter vingt-quatre sous qu'il donna au boulanger $\rangle^{55}$.

\section{Des pratiques ouvrières originales}

Si ces émeutes sont donc essentiellement populaires, il nous semble aussi que les ouvriers y réinvestissent des pratiques originales liées à leur identité forgée au travail. L'attachement à la qualification constitue une dimension fondamentale de cette identité, qui s'exprime notamment à travers le rapport aux outils cultivé par les ouvriers. Des coutumes leur rappellent la nécessité de les entretenir soigneusement et, au-delà, mettent en valeur la qualification qu'ils symbolisent. Les outils sont brandis, de manière menaçante, lors des émeutes : «Les ouvriers [de l'atelier de charité rassemblés devant le domicile d'un édile] étaient armés de leurs outils $»^{56}$, plus précisément de pelles ${ }^{57}$; le maçon meneur de l'émeute de 1789 exhibe « un grand ciseau propre à tailler du grison $»^{58}$. Si les outils sont utilisés comme armes, il nous semble aussi qu'ils symbolisent plus profondément une identité. Une double lecture comparable se retrouve dans les menaces proférées à leur encontre par les émeutiers qui s'efforcent d'imposer l'arrêt du travail. Un ouvrier charron explique que les émeutiers «prétendirent que les ouvriers des différentes boutiques les suivissent $[\ldots]$, sinon qu'ils prendraient les outils $»{ }^{59}$. Or, cette pratique apparaît dans les conflits du travail. De plus, un ouvrier des chantiers navals indique que, « lorsqu'une grande affluence d'hommes et de femmes vinrent crier qu'il fallait descendre et les accompagner à l'hôtel de ville, $[. .$.$] les calfats travaillant au même radoub se mirent à frapper$ avec leurs outils, manière ordinaire pour indiquer la cessation d'ouvrage et les rassemblements $\rangle^{60}$. Par ailleurs, la volonté d'imposer la cessation $\mathrm{du}$ travail est également issue des conflits du travail. Les compagnons imposent ainsi le respect de l'interdit jeté sur certaines boutiques. Un journalier embauché sur un chantier de construction rapporte ainsi que, le

(55) AD Loire-Atlantique, B 8746-IV, interrogatoire de J. Lédat.

(56) AD Loire-Atlantique, L 1440, déclaration de l'accusateur public du tribunal du district de Nantes, 7 décembre 1790.

(57) AD Loire-Atlantique, L 1440, déposition de J. Richard, 9 décembre 1790.

(58) AD Loire-Atlantique, B 8746-IV, interrogatoire de J. Lédat.

(59) AD Loire-Atlantique, L 1439, interrogatoire de C. Aubert, 8 septembre 1791.

(60) AD Loire-Atlantique, L 1439, interrogatoire de F. Hagas, 7 septembre 1791. 
5 septembre 1791, plusieurs « ouvriers de navires [...] s'étaient présentés pour entraîner avec eux tous ceux qui travaillaient à ladite construction et avaient menacé de les accabler de coups de pierres $\rangle^{61}$. Ainsi, lors des émeutes, les ouvriers recourent à des pratiques issues des conflits du travail. On a vu aussi qu'ils se mobilisaient par métier.

Si les ouvriers usent donc au cours des émeutes de pratiques originales, liées à leur identité spécifique forgée au travail, ils ne constituent pas pour autant un élément à part dans celles-ci, puisqu'ils partagent avec l'ensemble des émeutiers, outre les motifs, les pratiques et les valeurs populaires alors mises en œuvre. D'ailleurs, le caractère essentiellement populaire de ces émeutes et le réinvestissement dans celles-ci de certains aspects proprement ouvriers ne se contredisent pas, mais au contraire se confortent. En effet, pleinement insérés dans le peuple, les ouvriers se montrent très attachés à l'autonomie, valeur qui incite le peuple en général à agir de son propre chef en cas de défaillance des autorités, et que les ouvriers développent plus particulièrement au travail. De plus, en l'absence d'émeute au XVIIII siècle, le recours à la violence légitime a été entretenu par les ouvriers lors des conflits du travail, qui se multiplient dans la seconde moitié du siècle. Enfin, lors de ceux-ci, les ouvriers recourent à des pratiques partagées par l'ensemble du peuple, comme le montre le viol du domicile de l'édile chargé de l'atelier municipal.

\section{Conscience sociale et maturation révolutionnaire}

D'une manière générale, les affrontements révèlent en même temps qu'ils accentuent la conscience sociale et la politisation ${ }^{62}$.

"Elle lui reprocha d'avoir pris les armes contre sa patrie, [alors] qu'il ne devoit pas avoir d'autres intérest que les ouvriers »

Etant donné que les revendications, l'appartenance sociale, les pratiques et les valeurs des émeutiers impriment aux émeutes nantaises un caractère essentiellement populaire, la conscience sociale qui les anime l'est sans doute également. Celle-ci recouvre à nos yeux, à la fois, un sentiment d'appartenance et une perception des rapports sociaux. Les tensions avec les autorités, ainsi que la mise en cause des accapareurs et

(61) AD Loire-Atlantique, L 1439, interrogatoire de J. Ferouin, 8 septembre 1791. Bien sûr, cette pratique fournit aussi une excuse aux émeutiers arrêtés.

(62) Jean Nicolas (éd.), Mouvements populaires et conscience sociale, XVI ${ }^{e}-X I X^{e}$ siècles, Actes du colloque de Paris, 22-24 mai 1984, Paris, Maloine, 1985. 
des agioteurs nourrissent une conscience populaire, dans la mesure où le peuple soulevé se heurte alors à des éléments bourgeois. Accusé de « receler du bled $»^{63}$, l'échevin maltraité le 8 janvier 1789 est un négociant. Le 5 septembre 1791, un boulanger encourage les émeutiers en proclamant « qu'il étoit horrible que de f... négotiants voulussent faire de la monnoye avec du papier $»^{64}$. Or, la révolution municipale a permis à ces derniers de dominer la municipalité et la garde nationale ${ }^{65}$.

Cependant, mêlée à cette conscience populaire s'exprime parfois une conscience plus spécifiquement ouvrière. Des ouvriers se montrent hostiles à l'égard de gardes nationaux qui se trouvent être des maitres artisans : le 25 mai 1790, alors que plusieurs maîtres figurent dans les rangs de la garde nationale ${ }^{66}$, un ouvrier bonnetier s'écrie « qu'il falloit assomer tous ces habits bleus $\aleph^{67}$. Les ouvriers dénoncent plus particulièrement la répression : le 5 septembre 1791, un charpentier de navire est arrêté pour avoir dit « qu'il était surprenant de voir [les gardes nationaux] armés contre des ouvriers qui n'avaient point de défense $\|^{68}$; un garde national est intercepté par des ouvriers cordiers « qui lui dirent qu'ils ne vouloient point faire de mal, qu'il pouvoit se rendre où bon lui sembleroit, mais qu'il n'avoit pas besoin d'arme, [puis] lesdits ouvriers [...] lui ôtèrent son fusil et le jetterent $»^{69}$. Enfin, des ouvriers invitent leurs camarades de travail appelés, en tant que gardes nationaux, à réprimer les émeutes à refuser de servir, comme en témoigne ce dialogue entre deux fondeurs d'une grande manufacture métallurgique le 25 mai 1790 : «Ledit Journé demanda à lui déposant si, comme grenadier de la garde nationale, il prendroit les armes; à quoi ayant répondu qu'étant malade, il n'iroit pas, ledit Journé répliqua que s'il y allait, ce seroit pour se mettre du côté des paysans; que la femme de Journé ajouta qu'ils seroient bien bêtes d'y aller pour soutenir un tas de voleurs $»^{70}$.

Plus largement, des gardes nationaux sont accusés de trahir le peuple, tel ce cordonnier menacé par sa voisine :

(63) AM Nantes, FF 289, procès-verbal des échevins, 8 janvier 1789.

(64) AD Loire-Atlantique, L 1439, déposition de M. Cuissard, 16 septembre 1791.

(65) Olivier Pétré-Grenoullleau, Nantes, Plomelin, Palantines, 2003, p. 112.

(66) Leur condition est précisée dans leurs dépositions (AM Nantes, I 3-D 99).

(67) AM Nantes, I 3-D 99, déposition d'A. Debeluc, 26 mai 1790.

(68) AD Loire-Atlantique, L 1439, interrogatoire de M. Chartier, 6 septembre 1791. Rappelons le caractère ambigu du terme « ouvrier ».

(69) AD Loire-Atlantique, L 1439, déposition de F. Boisselot, 16 septembre 1791.

(70) AM Nantes, I 3-D 99, déposition de D. Manet, 27 mai 1790. Par ailleurs, précisons bien que le charpentier de navire, les cordiers et les fondeurs cités dans ce paragraphe sont des salariés et que le bonnetier est soit salarié, soit chambrelan. 
«La femme de Herse, charpentier, [...] vomi[t] une infinité d'injures au nommé Brousse, cordonnier, [...] pour avoir pris la veille les armes à l'effet de concourrir au rétablissement de la paix troublée par un attroupement considérable; elle lui reprocha d'avoir pris les armes contre sa patrie, [alors] qu'il ne devoit pas avoir d'autres intérest que les ouvriers et qu'il devoit lui-même se réunir pour faire tomber les cartes $\gg{ }^{71}$.

Précisons bien que, comme nous ignorons la condition sociale exacte des émeutiers désignés par leur seul métier ou par le terme général d'ouvrier, la conscience sociale qui s'exprime ici peut-être aussi bien largement populaire que spécifiquement ouvrière.

Une telle ambiguïté se retrouve lorsque les émeutiers se désignent eux-mêmes, manifestant alors un sentiment d'appartenance. En septembre 1789, Joseph Lédat, le meneur de l'émeute de janvier bientôt soupçonné d'être enrôlé dans un complot aristocratique, s'écrie : « Nous sommes bien des ouvriers dans Nantes, si nous voulions nous entendre, nous ferions diminuer le pain et le mangerions meilleur $\gg^{72}$. En fait, comme nous l'avons déjà suggéré plus haut en traitant du réinvestissement de pratiques ouvrières dans des émeutes populaires, ces manifestations d'une conscience largement populaire ou plus spécifiquement ouvrière ne nous semblent pas contradictoires mais complémentaires : il s'agit en effet de dimensions associées chez des ouvriers, qui restent insérés dans le peuple et participent précisément à des émeutes essentiellement populaires, et non de deux consciences juxtaposées qu'il serait artificiel et erroné de tenter de distinguer. Il est plus pertinent de s'intéresser à l'articulation du social et du politique dans la dynamique révolutionnaire, puis de s'interroger sur l'éventuel effet de cette expérience révolutionnaire sur l'identité sociale des ouvriers.

\section{Politisation et conscience révolutionnaire}

Si le peuple ne découvre pas la politique grâce à la Révolution française, il n'en est pas moins vrai que celle-ci s'accompagne d'une réelle politisation, qui passe par des références aux antagonismes, aux mesures, aux valeurs ou encore aux pratiques révolutionnaires. Dès janvier 1789 , dans une période où le conflit entre patriotes et aristocrates est exacerbé en Bretagne à cause de la réunion des États provinciaux, le meneur de l'émeute célèbre la baisse du prix du pain aux cris de « vive le roi! vive la liberté !

(71) AD Loire-Atlantique, L 1439, déposition de J. Legrand, 17 septembre 1791.

(72) AD Loire-Atlantique, B 8746-IV, déposition de P. Brinsouart, $1^{\text {er }}$ octobre 1789. 
vive le Tiers état! », et se montre hostile à la noblesse ${ }^{73}$. L'année suivante, lors de l'émeute de la foire nantaise, un ouvrier bonnetier estime que « les droits féodaux étant abolis $»^{74}$, l'octroi doit l'être aussi. Le 5 septembre 1791, en condamnant les gardes nationaux qui vont « tuer [leurs] frères », un tonnelier se montre sensible à cette valeur de la Révolution qu'est la fraternité $^{75}$. Enfin, le 6 décembre 1790, les ouvriers de l'atelier municipal menacent de «couper la teste du sieur Fourny et [de] la porter sur une picque, comme on avait fait à Paris $»^{76}$. Au-delà, la proclamation générale de droits conforte sans doute dans le peuple la conviction de la légitimité de ses revendications. La politisation se nourrit d'ailleurs de son articulation avec les questions sociales : aucun domaine n'échappe à la Révolution car les différents acteurs et groupes sociaux s'emparent des mesures et des valeurs révolutionnaires pour les appliquer à leurs propres préoccupations et revendications. L'articulation du social et du politique s'opère donc à travers l'intégration de références et de valeurs révolutionnaires à des combats sociaux ${ }^{77}$, et suscite ainsi la maturation d'une conscience révolutionnaire, qui correspond également à la conviction que la satisfaction des revendications devient possible. À cette maturation contribue aussi l'inscription des émeutes dans la dynamique révolutionnaire et, donc, dans les conflits de toutes natures qui la scandent.

L'articulation du social et du politique confère une certaine autonomie à la participation du peuple à la Révolution. En effet, le caractère essentiel pour lui des motifs sociaux suscite des tensions avec les autorités. À l'automne 1789, l'agitation provoquée par les difficultés frumentaires inquiète d'autant plus la nouvelle municipalité patriote qu'elle s'accompagne de propos hostiles à son égard ${ }^{78}:$ un portefaix affirme « qu'il est du côté de la noblesse, parce qu'il a couru la ville tout le jour sans pouvoir trouver de pain ${ }^{79}$; le meneur de la précédente émeute de janvier, accusé fin septembre d'être impliqué dans un complot aristocratique, est soupçonné

(73) AD Loire-Atlantique, B 8746-IV, interrogatoire de J. Lédat. Lors de l'émeute Réveillon, des émeutiers crient aussi « vive le Tiers État » (Georges RuDÉ, La foule dans la Révolution française, Paris, Maspéro, 1982, p. 53).

(74) AM Nantes, I 3-D 99, déposition de F. Huard, 29 mai 1790.

(75) AD Loire-Atlantique, L 1439, déposition d'E. Baudouin, 17 septembre 1791.

(76) AD Loire-Atlantique, L 1440, déposition de C. Raimbault, 9 décembre 1790.

(77) C'est ainsi que, dans la citation détachée plus haut, la conscience sociale est exprimée à travers un terme politique.

(78) Si, à Paris, l'hostilité soulevée par l'intransigeance aristocratique et la revendication frumentaire contribuent à déclencher les journées d'octobre, à Orléans la nouvelle municipalité affronte une émeute le 12 septembre 1789 (Georges LefEBVRE, Études orléanaises, t. 2, Subsistances et maximum, 1789-an IV, Paris, CHESR, XV, 1963, p. 31).

(79) AD Loire-Atlantique, B 8745-VI, déposition de J.-B. Eche, 13 octobre 1789. 
d'avoir proposé « de s'assembler pour faire une révolte contre la cherté de pain et pour f... malheur au Tiers état $\rangle^{80}$. À ses yeux, le Tiers correspond au parti patriote, qui se réduit en fait à l'élite portée au pouvoir par la révolution municipale. Celle-ci estime que l'obsession frumentaire du peuple le rend aveugle et versatile, et que sa politisation reste donc superficielle : par conséquent, elle interprète en termes de manipulation les émeutes de 1790 et 1791 . Certes, au début de la Révolution, la politisation populaire reste sans doute ténue et fragile, si bien qu'à Nantes comme ailleurs ${ }^{81}$, les tentatives de manipulation nobiliaires peuvent rencontrer un certain écho. De plus, dans le peuple, les niveaux de conscience et les orientations politiques sont hétérogènes. Toutefois, l'action populaire ne relève pas uniquement de la manipulation. Il nous semble que la politisation populaire est réelle dès 1789-1791 et qu'elle s'épanouit notamment à travers l'imprégnation révolutionnaire des questions sociales. De ce fait, la maturation révolutionnaire du peuple en général et des ouvriers en particulier se révèle en partie autonome et originale. Accélérée par ces affrontements, elle aiguise en retour la conscience sociale des émeutiers, consolidant leur conviction de la légitimité de leurs revendications et de leurs actions et, au-delà, avivant une lucidité qui peut porter certains à se défier tant de la bourgeoisie patriote que de l'aristocratie.

Accentuant la conscience sociale et la politisation et suscitant donc l'articulation du social et du politique, les émeutes alimentent la maturation d'une conscience révolutionnaire dans le peuple en général et chez les ouvriers en particulier. Pour ces derniers, cette maturation peut être définie plus précisément comme l'émergence progressive d'un ensemble de pratiques, de valeurs et de références issues tant de leur identité sociale et culturelle forgée au travail que de leur participation à la Révolution, lors de mobilisations largement populaires ou plus spécifiquement ouvrières. Mais, si pour les ouvriers, cette maturation présente quelques spécificités, cette conscience révolutionnaire ne leur est pas propre. Existe-t-il donc des domaines dans lesquels elle pourrait revêtir une dimension spécifiquement ouvrière?

\section{Des droits pour les ouvriers?}

Les ouvriers s'efforcent d'intégrer les principes révolutionnaires dans le domaine du travail.

(80) AD Loire-Atlantique, B 8746-IV, interrogatoire de J. Lédat.

(81) Par exemple, à Rennes lors de la journée des Bricoles, le 26 janvier 1789. 
«Voyant que l'on tolérait les sociétés des autres métiers, ils avaient cru pouvoir les imiter $\|^{82}$

Tout d'abord, de nombreux ouvriers sont convaincus que l'abolition des privilèges frappe les corporations et que la police du travail qu'elles mettent en œuvre meurt avec elles, si bien que les organisations ouvrières bénéficieraient alors d'une reconnaissance légale. Steven Kaplan a étudié pour Paris cette double question qui associe donc l'ambiguïté du sort réservé aux corporations d'une part, et les efforts entrepris par les compagnons pour bénéficier des droits révolutionnaires dans le domaine du travail, d'autre part ${ }^{83}$. À Nantes aussi, de nombreux ouvriers - non seulement les compagnons mais encore les portefaix qui, solidement organisés, combattent depuis un siècle pour imposer leur monopole sur les quais - sont persuadés que la Révolution liquide les privilèges et la police socio-économiques de l'Ancien Régime. On a vu qu'en novembre 1791, les autorités craignaient qu'une conjuration des compagnonnages tente de relancer l'émeute contre les billets de la caisse patriotique. En fait, l'interrogatoire d'un compagnon révèle que l'assemblée projetée des compagnonnages de tous les métiers a pour objet de les « faire consentir à ce que les compagnons maréchaux-ferrants qui, dans différentes villes du royaume, forment des sociétés de compagnons du devoir, en formassent également une en cette ville de Nantes, reconnue et avouée par les autres avec lesquels il leur était intéressant de fraterniser parce que dans le besoin les unes et les autres assistent et secourent réciproquement leurs différents membres $»^{84}$. Ce compagnon espère aussi bénéficier de la tolérance des autorités. Si celles-ci ont pu un temps se montrer tolérantes, partageant en fait la conviction des ouvriers selon laquelle les droits révolutionnaires s'appliquaient au domaine social ${ }^{85}$, l'adoption de la loi Le Chapelier en juin 1791 a mis un terme à cette période confuse : l'édile qui interroge ce maréchal-ferrant lui réplique « qu'il a tort d'alléguer cette prétendue tollérance qui n'a jamais eu lieu », et est définitivement écartée par « la nouvelle constitution ${ }^{86}$. Cette conviction de la reconnaissance

(82) AD Loire-Atlantique, L 2073, interrogatoire de J. Potel, 25 novembre 1791.

(83) Il écrit notamment que «pour les ouvriers, aucun des droits révolutionnaires n'était aussi important que la liberté de s'assembler » (Steven KAPLAN, La fin des corporations, Paris, Fayard, 2001, p. 424).

(84) AD Loire-Atlantique, L 2073, interrogatoire de J. Potel, 25 novembre 1791.

(85) Par exemple, en 1790, un commissaire de police répond positivement aux portefaix venus le solliciter pour défendre le monopole que s'arroge leur organisation.

(86) AD Loire-Atlantique, L 2073, interrogatoire de J. Potel, 25 novembre 1791. 
- du moins l'espoir de la tolérance - des organisations ouvrières repose aussi sur la convergence, aux yeux des ouvriers, entre leurs pratiques traditionnelles et les valeurs révolutionnaires : le compagnon maréchal-ferrant présente l'assemblée des compagnonnages comme une fraternisation.

Cette concordance entre la solidarité pratiquée par les ouvriers et la fraternité exaltée par la Révolution, d'une part, et la conviction que les principes révolutionnaires doivent s'appliquer au domaine du travail, d'autre part, amènent certains ouvriers à mépriser la loi Le Chapelier, à l'instar d'un compagnon maréchal-ferrant qui, lors de son arrestation, proclame « que la liberté étoit en France et qu[e] personne [ne peut donc] les empescher de faire le compagnonage et qu'il existera toujours $\rangle^{87}$. Peutêtre qu'à l'instar des combats démocratiques menés au nom des droits de l'homme contre le suffrage censitaire, l'esclavage ou même le rachat des droits féodaux, ces ouvriers attendent la pleine actualisation des principes révolutionnaires. $\mathrm{Si}$, comme celui des citoyennes pour les droits civiques, ce combat n'aboutit pas, il n'en contribue pas moins à aiguiser la conscience révolutionnaire de ceux qui le mènent. Cependant, cette réaction démocratique aux contradictions politiques et sociales n'a rien d'automatique. En effet, comme les tensions agraires ou la disette, elles peuvent aussi nourrir un sentiment antirévolutionnaire.

Néanmoins, ce combat propre aux ouvriers s'accompagne bien de l'aiguisement d'une conscience sociale particulière et d'une politisation qui passe elle-même par l'intégration des principes révolutionnaires à une lutte sociale spécifique. La perception de la condition ouvrière en termes de droits - déjà apparue lors de conflits du travail à la fin de l'Ancien Régime à travers l'emploi du vocabulaire politique des Lumières lors de démarches juridiques ${ }^{88}$ - pourrait favoriser l'émergence d'une conscience ouvrière, d'autant plus que cette aspiration à la reconnaissance des organisations ouvrières anime les ouvriers de tous les métiers, par-delà la diversité des formes d'organisation et leur degré d'institutionnalisation ${ }^{89}$. Toutefois la défense de leurs organisations accentue plutôt la conscience professionnelle des ouvriers, étant donné que ces organisations cristallisent une identité fondée sur la qualification, donc le métier. Au sein du monde ouvrier, l'esprit de corps professionnel se renforce d'ailleurs au

(87) AD Loire-Atlantique, L 2074, procès-verbal du commissaire Albert, 13 juin 1792.

(88) Michael Sonenscher, Work and Wages. Natural Law, Politics and the Eighteenth-century French Trades, Cambridge, CUP, 1989.

(89) Certes, elle ne mobilise pas les journaliers dépourvus de qualification comme d'organisation. 
tournant des $\mathrm{XVIII}^{\mathrm{e}}$ et $\mathrm{XIX}{ }^{\mathrm{e}}$ siècles, en fait au début de l'industrialisation. Il apparaît donc qu'un combat similaire, voire commun ne donne pas nécessairement naissance à une conscience collective.

\section{«Ces ouvriers [...] prétendent que la commune doit continuellement leur fournir du travail $»^{90}$}

Menacés de renvoi, les ouvriers employés par l'atelier municipal de charité se rassemblent à la fin de l'année 1790 au domicile de l'édile qui en est responsable. On ignore le métier originel de ces « ouvriers et manœuvres », comme les désigne l'accusateur public ${ }^{91}$. En violant le domicile de cet édile, ils usent de pratiques employées pour la traque des accapareurs. De plus, comme d'autres ouvriers lors d'émeutes populaires, ils brandissent leurs outils, geste qui, sur le plan symbolique, nous semble issu de l'identité ouvrière forgée au travail. Enfin, l'inscription de cette manifestation dans la Révolution apparaît clairement lorsque les ouvriers envisagent d'imiter les insurgés parisiens en fichant la tête de leur victime au bout d'une pique.

Au-delà, il semble que les ouvriers étayent la revendication à conserver leur travail de la référence à un droit à l'emploi. Si l'appel à un tel droit n'est pas nouveau ${ }^{92}$ et s'il peut s'inscrire, à l'instar de la revendication frumentaire, dans l'économie morale du peuple, comme le suggère l'association de la revendication du pain et de celle du travail lors de l'émeute de janvier 1789, il se peut que cette revendication ancienne et la conviction de sa légitimité soient renforcées par la proclamation générale de droits lors de la Révolution. Même si elle ne se fonde pas sur un principe nettement affirmé comme le droit d'association, la conviction de l'existence de ce droit à l'emploi s'apparente à celle de la reconnaissance des organisations ouvrières, dans la mesure où elle procède aussi de l'intégration de valeurs révolutionnaires à un combat social. De ce fait, ce combat contribue à la maturation d'une conscience révolutionnaire. De plus, à l'instar

(90) AD Loire-Atlantique, L 1440, déclaration de l'accusateur public du tribunal du district de Nantes, 7 décembre 1790.

(91) Si certains sont sans doute des journaliers, d'autres sont probablement des ouvriers qualifiés privés d'ouvrage par la crise économique de la seconde moitié des années 1780.

(92) Charles Engrand montre la vigueur de la conviction du droit à l'emploi dans le monde du travail amiénois sous l'Ancien Régime. En 1789, des travailleurs employés par l'atelier municipal de charité exigent « du travail ou du pain ». Charles Engrand, «Conscience populaire et droit à l'emploi : l'exemple d'une grande ville manufacturière, Amiens ( $\mathrm{XVI}^{\mathrm{e}}-\mathrm{XVIII}{ }^{\mathrm{e}}$ siècle) », dans Philippe Guignet (éd.), Le peuple des villes dans l'Europe du Nord-Ouest (fin du Moyen Age-1945), Lille, CRHENO, 2003, vol. 2, p. 261. 
de l'exigence opiniâtre de l'application du droit d'association au domaine du travail, la revendication du droit à l'emploi pourrait nourrir l'espoir démocratique d'une actualisation élargie des principes révolutionnaires, qui, elle - et contrairement au combat pour la reconnaissance des organisations ouvrières - recevrait une consécration dans le fameux article 21 de la Déclaration de $1793^{93}$. Mais, par ailleurs, si les ouvriers y sont sans doute très attachés et s'ils en sont les principaux défenseurs, cette revendication d'un droit au travail est probablement plus largement populaire ${ }^{94}$. Dès lors, même si elle est portée par l'ensemble des ouvriers - y compris les journaliers, à l'inverse du combat pour la défense des organisations ouvrières - cette revendication ne contribue pas nécessairement à l'émergence d'une conscience ouvrière particulière.

Ces combats pour la reconnaissance de leurs organisations et le droit au travail nourrissent chez les ouvriers qui les mènent la maturation d'une conscience révolutionnaire, parce qu'ils se fondent sur l'intégration de principes et de valeurs révolutionnaires à des luttes sociales. Mais si ces combats sont menés par des ouvriers, le premier peut accentuer surtout leur conscience professionnelle, tandis que le second reste peutêtre largement populaire. Par conséquent, il est impossible de conclure que la revendication par les ouvriers de droits lors de la Révolution donne nécessairement naissance à une conscience ouvrière.

Si, lorsqu'il signale à la municipalité la préparation d'une nouvelle émeute par les compagnons du devoir, le patron d'une grande manufacture textile écrit qu'il « ne croi[t] pas qu'une émeute populaire puisse devenir bien dangereuse $»^{95}$, les manifestations qui se succèdent à Nantes de 1789 à 1791 contribuent grandement au développement de la participation à la Révolution française du peuple en général et des ouvriers en particulier, car elles accentuent tant la conscience sociale que la politisation. Importante et variée, la participation des ouvriers à la Révolution s'inscrit donc souvent dans celle de l'ensemble du peuple. De plus, lorsqu'elle

(93) Jean-Pierre Gross, Égalitarisme jacobin et droits de l'homme, 1793-1794. La Grande famille et la Terreur, Paris, Arcantères, 2000, chap. VII (1997).

(94) Les petits maîtres amiénois déclassés sous l'Ancien Régime comme les artisans parisiens hostiles à la concentration de la production des fournitures de guerre sous la Révolution (Haïm Burstin, « Problèmes du travail à Paris sous la Révolution », Revue d'Histoire moderne et contemporaine, 1997-4, p. 659) font appel au droit au travail.

(95) AD Loire-Atlantique, L 2073, lettre de L. Saget à D. Kervégan, 24 novembre 1791. 
porte sur des questions spécifiquement ouvrières, elle conforte parfois une conscience essentiellement professionnelle. Par ailleurs, articulant le social et le politique, cette participation suscite chez ces ouvriers la maturation d'une conscience révolutionnaire, que nous avons définie comme l'émergence progressive d'un ensemble de pratiques, de valeurs et de références issues de leur identité sociale et culturelle comme de leur participation à la Révolution. Au-delà, cette conscience révolutionnaire correspond également, non seulement à la conviction de la possibilité nouvelle de satisfaire les aspirations et les revendications, mais encore à l'approfondissement du sentiment - si fortement mobilisateur - de leur légitimité; mais cela n'est pas propre aux ouvriers. Dès lors, si nous pouvons donc souligner le caractère remarquable de l'expérience révolutionnaire des ouvriers nantais, nous devons préciser que la maturation d'une conscience révolutionnaire ne leur est pas propre, même si elle présente, dans leur cas, des aspects originaux. Si elle a pu renforcer leur identité commune à travers le combat pour le développement de droits dans le domaine du travail, elle ne donne pas naissance chez eux à une conscience collective et spécifique.

Samuel Guichet eau

Chercheur associé au Cerhio-Rennes 2 14 rue Jean Bart 44220 Couëron samuel.guicheteau@orange.fr 